\title{
Fault Mechanism of Fiber Optical Current Transformer Based on Signal Processing Method
}

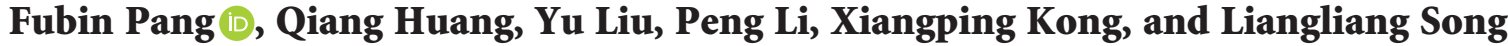 \\ State Grid Jiangsu Electric Power Co., Ltd. Research Institute, Nanjing 211103, China \\ Correspondence should be addressed to Fubin Pang; pangfubin2006@163.com
}

Received 27 January 2020; Revised 21 March 2020; Accepted 24 March 2020; Published 15 April 2020

Academic Editor: Gaetano Zizzo

Copyright (c) 2020 Fubin Pang et al. This is an open access article distributed under the Creative Commons Attribution License, which permits unrestricted use, distribution, and reproduction in any medium, provided the original work is properly cited.

\begin{abstract}
The fiber optical current transformer (FOCT) with sine wave modulation has been widely used in the DC transmission project, while its fault rate is much higher than that of an electromagnetic current transformer, which seriously threatens the safe operation of power grid. In order to investigate the fault mechanism of FOCT, the output signal model of the FOCT photodetector is established, and its basic methods of modulation and demodulation are analyzed to explore the influencing factors of the signal processing method used in the calculation of measured current. It is found that the reduction of the second harmonic component in the photodetector output is the root cause of FOCT fault, and the optical path, modulation signal amplitude, and measured current are important factors affecting the second harmonic component. The influencing law of three factors on the second harmonic component is calculated by simulation and the characteristic differences between them are given. Finally, the fault simulation test of FOCT light intensity and a modulation circuit were carried out in the laboratory, and the results verify the correctness of theoretical analysis. The research in this paper provides reference for fault analysis and reliability improvement of FOCT.
\end{abstract}

\section{Introduction}

In recent years, the construction of an ultrahigh-voltage direct current (UHVDC) transmission project has been speeding up. There are more than 30 converter stations in operation in China, which constitute the backbone network of long-distance and cross-regional power transmission [1-3]. As the main sensor of analog data acquisition for control and protection system, the DC current transformer in DC engineering has a direct impact on the safety and stability of the power grid, and the research on its fault mechanism has been also increasingly concerned.

The DC current transformer can be divided into two types according to its working principle: zero-flux type and photoelectric type $[4,5]$, and the latter includes active and passive electronic current transformers, whose typical representatives are photoelectric current transformers based on shunt principle and fiber optical current transformer (FOCT) based on Faraday magneto-optical effect, respectively [6-8]. Many researchers have analyzed the abnormal operation characteristics of different types of DC current transformers, which have received extensive attention. The Bayesian method is used to make a judgment of operating state of power supply lasers of the photoelectric current transformer through measuring the current of MU's power source [9]. The methods of condition monitoring and fault diagnosis in FOCT using the model based on Allan variance is proposed, and experiment results show that the diagnostic methods are accurate and effective to identify fault features [10]. The real-time dynamic simulation model of FOCT is established, the influences of temperature on the Verdet constant and $(\lambda / 4)$ wave plate of optical fiber are analyzed, the comprehensive error simulation analysis and experimental verification of FOCT are given when the temperature fluctuates, and the specific measures of temperature compensation are proposed $[11,12]$. According to the topology of converter station, the configuration matrix of the DC transformer is built, the real-time diagnosis method of transformer abnormal state is proposed by using causal network, and the simulation of transformer abnormal state proves the effectiveness of the method [13]. The influencing factors analysis on the detector output signal of the fiber 
optic current transformer is investigated by establishing the detector output of FOCT, and calculation and simulation are carried out to study the influencing law of the factors [14].

However, the aforementioned research mainly focuses on the analysis of DC current transformer fault performance, or its measurement error under a certain influencing factor (such as temperature), while little has been done to reveal its fault mechanism based on its signal processing method. In this paper, the output model of the FOCT photodetector with sine modulation is established to investigate its fault mechanism, and the factors that affect its signal processing method are further analyzed to explore the cause of the fault. It is found that the second harmonic component of the photodetector output is the key parameter to calculate the measured current, and the influences of the optical path, modulation signal amplitude, and measured current on the second harmonic component are calculated by simulation and verified by experiment.

\section{FOCT Working Principle}

FOCT measures the primary current by detecting the interference phase difference of two polarized light beams under Faraday magneto-optical effect $[15,16]$. The schematic diagram in Figure 1 shows the working principle of FOCT. Driven by the LED source driving circuit, the light signal from LED source enters the polarizer to form linearly polarized light after passing through the coupler. After the splitting of $45^{\circ}$ fusion, the linearly polarized light turns into two perpendicular linearly polarized lights on $x$ - and $y$-axis, which still keep orthogonal to each other after phase modulation and transmission in polarization-maintaining fiber. Passing through the $(\lambda / 4)$ wave plate, the two beams of orthogonal polarized lights are respectively converted into left-handed and right-handed circularly polarized lights and enter the sensing loop, where they generate Faraday phase shift proportional to the measured primary current under the action of the Faraday magneto-optical effect. At the reflector, the polarization states of the two beams are interchanged, and the Faraday magneto-optical effect of the magnetic field is experienced again in the sensing loop. The phase shift between the two circularly polarized light beams is doubled, and the two beams are recovered to perpendicular lights after the wave plate, which then interfere with each other and enter the photodetector after the $45^{\circ}$ fusion point. Finally, the light intensity with current information is sent to the detector through the coupler, amplified by the amplifier, converted by $A / D$ conversion, and processed by signal processing unit to calculate the primary current.

During the whole transmission, the light signal is initially transmitted clockwise, and the left-handed and right-handed circularly polarized lights produces Faraday phase shift, respectively; at the reflector, the light signal becomes counterclockwise, and the left-handed and right-handed circularly polarized lights produce the same Faraday phase shift. Therefore, there are two Faraday phase shifts at the polarizer after interference, and light intensity detected by the photodetector can be expressed as

$$
P(t)=0.5 K L P_{0}(1+\cos (\Delta \theta)),
$$

where $K$ is the photoelectric conversion coefficient of the photodetector, $L$ is the transmission loss of the optical path, $P_{0}$ is the light intensity generated by the LED source, and $\Delta \theta=4 N I V$ is the Faraday phase shift $(N, I$, and $V$ represent the number of sensing loops, magnitude of the primary current, and Verdet constant of the fiber, respectively).

It can be seen from the abovementioned process that when the light signal returns to the coil from the reflector, the incident and reflected polarized light exchanges the polarization state so that the influences of additional linear birefringence are eliminated. Meanwhile, there is no reciprocity for Faraday magneto-optical effect and the phase shift is doubled. Therefore, FOCT with this reflective interference structure can not only reduce the influence of linear birefringence but also double the Faraday magnetooptical effect and improve the sensitivity of measurement.

\section{Analysis on FOCT Fault Mechanism}

3.1. Output Signal Model of FOCT Photodetector. It can be derived from equation (1) that the output signal of the FOCT photodetector cannot distinguish current direction and has low sensitivity. Therefore, it is necessary to apply phase modulation to the polarized lights of two beams by using a phase modulator. Generally, there are two kinds of phase modulation, namely, sine wave modulation and square-wave modulation [17, 18]. For sine wave modulation, a PZT (piezoelectric ceramic) phase modulator is usually inserted into the fiber coil considering the cost, and the sinusoidal phase modulation is generated for the light signal in the fiber ring by applying sinusoidal signal to PZT. The square-wave modulation generally adopts the photoelectric modulation mechanism such as lithium niobate crystal, and its modulation frequency reaches to thousands of $\mathrm{MHz}$, which can achieve better measurement accuracy. However, the sine wave modulation is widely used in converter stations in China for the advantage of low cost, no need of field optical fiber fusion, easy installation, and sufficient measurement accuracy for engineering requirements.

For FOCT with sine wave modulation, the modulation signal is given by

$$
\phi(t)=\phi_{\omega} \sin \omega t
$$

where $\phi_{\omega}$ and $\omega$ represent the amplitude and angular frequency of the modulation signal. Let $\tau$ denote the time delay in the optical fiber, and the phase modulation generated by the superposition of forward-transmitting and reflected beams can be understood from the following:

$$
\Delta \phi=\phi(t)-\phi(t-\tau)=2 \phi_{\omega} \sin \left(\frac{\omega \tau}{2}\right) \cos \omega\left(t-\frac{\tau}{2}\right)
$$

By introducing equations (2) and (3) into equation (1), the output of the photodetector is given by

$$
P(t)=0.5 K L P_{0}\left\{1+\cos \left[\Delta \theta+2 \phi_{\omega} \sin \left(\frac{\omega \tau}{2}\right) \cos \omega\left(t-\frac{\tau}{2}\right)\right]\right\} .
$$




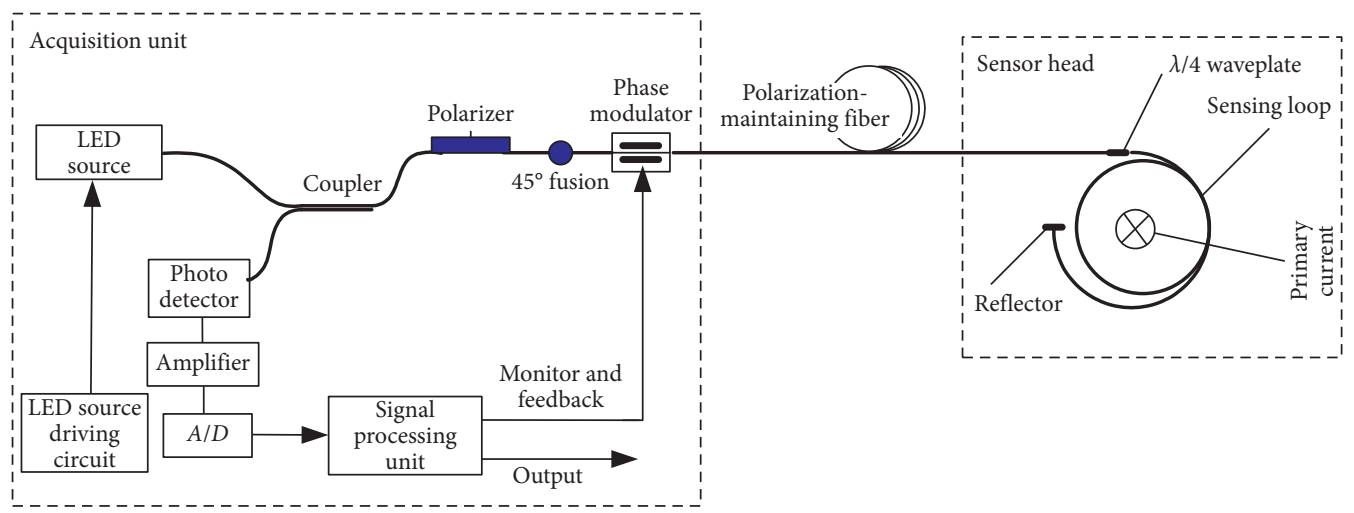

Figure 1: Schematic diagram of FOCT working principle.

Let $\phi_{e}=2 \phi_{\omega} \sin (\omega \tau / 2)$, and define it as the modulation factor, $t^{\prime}=t-(\tau / 2)$; then, equation (4) can be written as

$$
\begin{aligned}
P(t)= & 0.5 K L P_{0}\left\{1+\cos \left[\Delta \theta+\phi_{e} \cos \omega t^{\prime}\right]\right\} \\
= & 0.5 K L P_{0}\left[1+\cos \Delta \theta \cos \left(\phi_{e} \cos \omega t^{\prime}\right)\right. \\
& \left.-\sin \quad \Delta \theta \quad \sin \left(\phi_{e} \cos \omega t^{\prime}\right)\right] .
\end{aligned}
$$

According to the expansion form of Bessel function,

$$
\left\{\begin{array}{l}
\cos (z \cos \theta)=J_{0}(z)+2 \sum_{n=1}^{\infty}(-1)^{n} J_{2 n}(z) \cos (2 n \theta), \\
\sin (z \cos \theta)=-2 \sum_{n=1}^{\infty}(-1)^{n} J_{2 n-1}(z) \cos [(2 n-1) \theta],
\end{array}\right.
$$

where $J_{2 n}(z)$ is the 2 nd order Bessel function of $z$. The output of the photodetector can be expanded as follows:

$$
\begin{aligned}
P(t)= & 0.5 K L P_{0}\left\{1+\left[J_{0}\left(\phi_{e}\right)+2 \sum_{n=1}^{\infty}(-1)^{n} J_{2 n}\left(\phi_{e}\right) \cos \left(2 n \omega t^{\prime}\right)\right] \cos \quad \Delta \theta+2 \sum_{n=1}^{\infty}(-1)^{n} J_{2 n-1}\left(\phi_{e}\right) \cos \left((2 n-1) \omega t^{\prime}\right) \sin \Delta \theta\right\} \\
= & 0.5 K L I_{0}\left\{1+\left[J_{0}\left(\phi_{e}\right)-2 J_{2}\left(\phi_{e}\right) \cos 2 \omega t^{\prime}+2 J_{4}\left(\phi_{e}\right) \cos 4 \omega t^{\prime}+\cdots\right] \cdot \cos \Delta \theta\right. \\
& \left.+2\left[-J_{1}\left(\phi_{e}\right) \cos \omega t^{\prime}+J_{3}\left(\phi_{e}\right) \cos 3 \omega t^{\prime}+\cdots\right] \sin \Delta \theta\right\},
\end{aligned}
$$

where $J_{n}\left(\phi_{e}\right)$ is given by

$$
J_{n}\left(\phi_{e}\right)=\sum_{k=0}^{\infty} \frac{(-1)^{k}\left(\phi_{e} / 2\right)^{n+2 k}}{k !(n+k) !}, \quad k \geq 0 .
$$

3.2. Signal Processing Method. As reported in equation (7), the output of the photodetector can be regarded as the sum of infinite harmonic, while each harmonic carries Faraday phase shift $\Delta \theta$ information proportional to the measured current. In order to calculate the measured current, the fundamental and second harmonic components are taken as

$$
\begin{aligned}
& P_{1}=-K L P_{0} J_{1}\left(\phi_{e}\right) \sin \Delta \theta, \\
& P_{2}=-K L P_{0} J_{2}\left(\phi_{e}\right) \cos \Delta \theta .
\end{aligned}
$$

Generally, the Faraday phase shift $\Delta \theta$ is small, and thus, the fundamental component $P_{1}$ is far less than the second harmonic component $P_{2}$. In order to obtain higher sensitivity, the amplitude ratio of the second harmonic component $P_{2}$ to the fundamental $P_{1}$ can be obtained as follows:

$$
\frac{P_{2}}{P_{1}}=\frac{J_{2}\left(\phi_{e}\right)}{J_{1}\left(\phi_{e}\right)} \cot \quad \Delta \theta
$$

Finally, the measured current is obtained by calculating the Faraday phase shift $\Delta \theta$ :

$$
I=\frac{\Delta \theta}{4 V N}=\frac{1}{4 V N} \operatorname{arccot} \frac{P_{2} J_{1}\left(\phi_{e}\right)}{P_{1} J_{2}\left(\phi_{e}\right)} .
$$

It can be derived from equation (12) that the fundamental and second harmonic components should be extracted for signal processing for FOCT with sine wave modulation. However, the real-time calculation is relatively complicated to obtain the measured current. Since the Faraday phase shift $\Delta \theta$ is small and can be expressed as

$$
\Delta \theta=\arctan \frac{P_{1} J_{2}\left(\phi_{e}\right)}{P_{2} J_{1}\left(\phi_{e}\right)}
$$


The small angle approximation is usually adopted to save calculation to meet the requirements of real-time measurement:

$$
I=\frac{1}{4 V N} \arctan \frac{P_{1} J_{2}\left(\phi_{e}\right)}{P_{2} J_{1}\left(\phi_{e}\right)} \approx \frac{P_{1} J_{2}\left(\phi_{e}\right)}{4 V N P_{2} J_{1}\left(\phi_{e}\right)} .
$$

3.3. Fault Mechanism Analysis. Equation (12) indicates that the calculation of the measured current is related to the amplitude of the fundamental component $P_{1}$, second harmonic component $P_{2}$, and the first and second order Bessel functions of the modulation factor $\phi_{e}$. The sine wave modulation is completed by the analog circuit, which brings great electronic noise to FOCT. In order to ensure the accuracy of measured current calculation, certain signal-tonoise ratio (SNR) is required for signal processing. Therefore, the amplitude of the effective signal should be judged and the fault alarm signal should be sent to indicate the abnormal current working status of FOCT when this signal is too low to reach enough SNR.

Combining equations (9) and (10), it can be seen that among the four factors $P_{1}, P_{2}, J_{1}\left(\phi_{e}\right)$, and $J_{2}\left(\phi_{e}\right)$ affecting the measured current in equation (12), the first and second order Bessel functions of the modulation factor are contained in the fundamental and the second harmonic component. Besides, the fundamental harmonic component is far less than the second due to the small value of Faraday phase shift $\Delta \theta$; thus, the second harmonic component is the optimum signal to evaluate the working status of FOCT. In fact, it can be derived from equation (10) that the second harmonic component $P_{2}$ is not only proportional to the photoelectric conversion coefficient $K$, the transmission loss $L$, and light intensity $P_{0}$ but also closely related to the modulation factor $\phi_{e}$ and Faraday phase shift $\Delta \theta$. It does not only represent the working state of the optical path but also reflect the indicators such as modulation circuit and the Faraday phase shift $\Delta \theta$. Since the number of sensing loops $N$ and the Verdet constant $V$ are unchanged during the stable operation, the measured current $I$ is the indicator of $\Delta \theta$. Therefore, the optical path, modulation signal amplitude, and measured current are three main factors affecting the second harmonic component. The change of these factors may reduce the amplitude of the second harmonic component $P_{2}$ and further cause FOCT fault alarm once $P_{2}$ is less than the threshold value required by SNR for signal processing.

\section{Numerical Calculation}

4.1. Simulation Model. In order to analyze the influences of the optical path and modulation circuit on the second harmonic and further explore mechanism of FOCT fault, the changing rule of the second harmonic component under different optical path and modulation circuit parameters are calculated. In the simulation model, the product of $K, L$, and $P_{0}$ is $K L P_{0}=2 \mathrm{~V}$ for the optical path; for the modulation circuit, the length of optical fiber is $150 \mathrm{~m}$, corresponding to the time delay $\tau=1 \mu$ s for the round trip, and the amplitude and angular frequency of modulation signal are $\phi_{\omega}=4 \mathrm{rad}$ and $\omega=5.23 \times 10^{5}(\mathrm{rad} / \mathrm{s})$, respectively; thus, the modulation factor $\phi_{e}=2.07 \mathrm{rad}$; for the measured current, $N=20$, $I=2000 \mathrm{~A}, \quad V=0.95(\mathrm{rad} /(\mathrm{T} \cdot \mathrm{m}))=1.2 \times 10^{-6}(\mathrm{rad} / \mathrm{A})$, and $\Delta \theta=0.192 \mathrm{rad}$.

4.2. Influences of Optical Path Parameters. According to the simulation parameters in Section 4.1, the influences of the optical path parameters on the output of FOCT photodetector are compared when $K L P_{0}=2 \mathrm{~V}$ and $K L P_{0}=1 \mathrm{~V}$, and the results are shown in Figure 2.

The results in Figure 2 show that when the product of optical path parameters $K L P_{0}$ reduces from $2 \mathrm{~V}$ to $1 \mathrm{~V}$, the peak of the photodetector output is also reduced from $2 \mathrm{~V}$ to $1 \mathrm{~V}$, indicating that there is a positive proportional relationship between the two. The groove of the output peak is generated as the output signal is $0.5 K L P_{0}\{1+\cos$ $\left.\left[\Delta \theta-\phi_{e}\right]\right\}$ when $\omega t^{\prime}=\pi$ in equation (5), and the amplitude of second half cycle goes through that of the first half.

Figure 3 shows the comparison results of second harmonic components under the two parameters. Obviously, the amplitude of the second harmonic is also proportional to the product of the optical path parameters $K L P_{0}$. Therefore, the photodetector output signal and its second harmonic component containing Faraday phase shift information will also be reduced when the optical path attenuation is large in case of defective fiber splicing process, fiber fusion joint contamination, and loose or even broken optical fiber connection. If the amplitude of the second harmonic component is lower than the requirement of SNR, FOCT cannot proceed signal demodulation for the measured current and a fault alarm will be generated. The optical path problems can be controlled by strengthening management and control of fusion technology in factory construction process and regular maintenance, but there are still some hidden dangers in the fusion of optical fiber due to the poor field environmental conditions such as dust, temperature, and humidity.

\subsection{Influences of Modulation Circuit Parameters.} Equation (7) shows that when the Faraday phase shift is determined, the amplitude of each harmonic output of FOCT photodetector is closely related to the value of Bessel function of the modulation factor. In order to intuitively display the relationship between the amplitude of each harmonic and the modulation factor, the value of $0-4$ th order Bessel function is calculated by simulation and the results are shown in Figure 4.

As shown in Figure 4, except that the 0th order Bessel function reaches the maximum value when $\phi_{e}$ is 0 , the other order Bessel functions have a value of 0 when $\phi_{e}$ is 0 and represent a trend of oscillation attenuation with the increase of $\phi_{e}$. In addition, the lower order Bessel function first reaches the maximum amplitude earlier than the higher order with the increase of $\phi_{e}$, and the maximum amplitude of each order Bessel function also gradually decreases. Therefore, in order to ensure the sensitivity of signal 


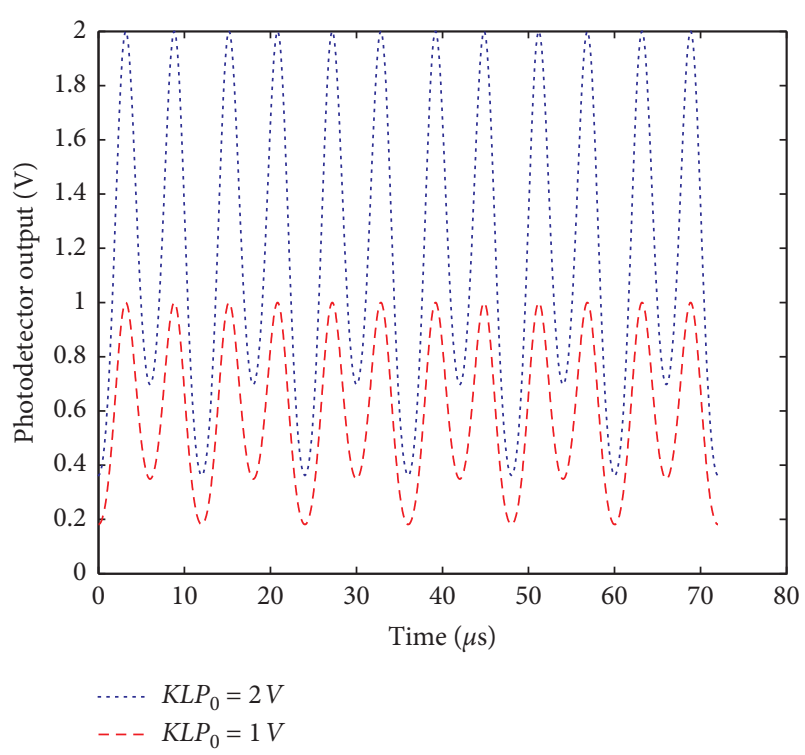

FIGURE 2: Comparison of the photodetector output under different optical path parameters.

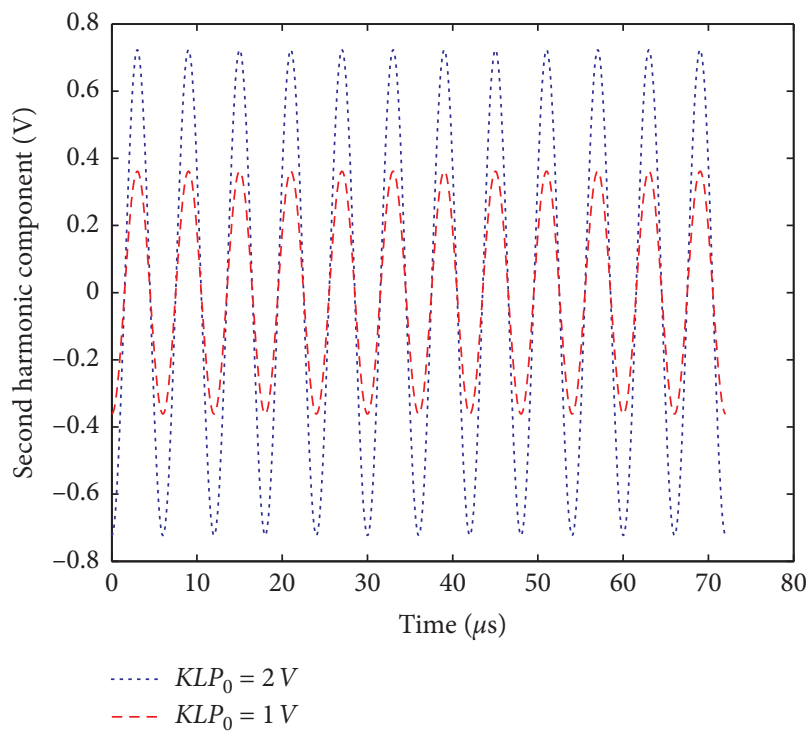

Figure 3: Comparison of second harmonic components under different optical path parameters.

processing, the fundamental and second harmonic components should be selected as the suitable signal. It can be seen that when $\phi_{e}=1.8 \mathrm{rad}$ and $\phi_{e}=3.1 \mathrm{rad}$, the fundamental and second harmonic components reaches the maximum, respectively, which is also the common value zone of the modulation factor of the PZT phase modulator.

In practical engineering applications, the transmission delay $\tau$ of the optical fiber and the angular frequency $\omega$ of the modulation signal are fixed parameters when FOCT is installed, and only the amplitude $\phi_{\omega}$ of modulation signal is easily affected by the long-distance modulation cable because of the distribution parameter of the long cable, resulting in the amplitude attenuation of the modulation

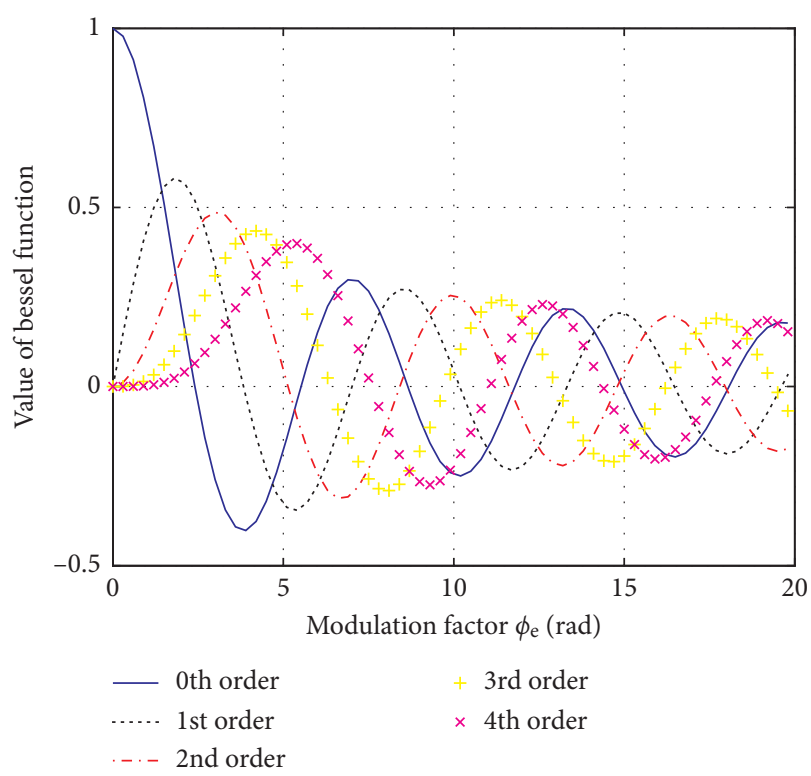

Figure 4: The relationship between the value of Bessel function and the modulation factor $\phi_{e}$.

factor $\phi_{e}$ and further affect the FOCT output. Figure 5 shows the FOCT photodetector output when $\phi_{\omega}$ is $4 \mathrm{rad}, 2 \mathrm{rad}$, and $1 \mathrm{rad}$, respectively.

Obviously, when $\phi_{\omega}$ is $4 \mathrm{rad}, 2 \mathrm{rad}$, and $1 \mathrm{rad}$, respectively, the maximum value of the photodetector output keeps $2 \mathrm{~V}$, while the minimum value is $0.36 \mathrm{~V}, 1.34 \mathrm{~V}$, and $1.75 \mathrm{~V}$, respectively, indicating a decrease of the output range with the reduction of $\phi_{\omega}$. This is because when $\phi_{\omega}$ declines, the modulation factor $\phi_{e}$ also decreases, resulting in the increase of the minimum value $0.5 K L I_{0}\left(1+\cos \left(\Delta \theta+\phi_{e}\right)\right)$ of the photodetector output signal in equation (5). The groove value $0.5 K L P_{0}\left\{1+\cos \left[\Delta \theta-\phi_{e}\right]\right\}$ for different modulation signal amplitudes also increases gradually, which is caused by the decrease of $\phi_{e}$.

Figure 6 is the comparison of second harmonic components under different modulation signal amplitudes. When $\phi_{\omega}$ is $4 \mathrm{rad}, 2 \mathrm{rad}$, and $1 \mathrm{rad}$, the peak value of second harmonic components is $0.71 \mathrm{~V}, 0.22 \mathrm{~V}$, and $0.05 \mathrm{~V}$, representing a fast decreasing trend.

This is because the decrease of $\phi_{\omega}$ leads to the drop of $\phi_{e}$, which reduces the amplitude of $J_{2}\left(\phi_{e}\right)$ in the second harmonic component (as shown in Figure 4, there is a positive proportional relationship between $\phi_{e}$ and its 2nd order Bessel function $J_{2}\left(\phi_{e}\right)$ when $\phi_{e}$ is no greater than $\left.3.1 \mathrm{rad}\right)$. Obviously, when the amplitude attenuation of the modulation signal causes the amplitude of the second harmonic component to be smaller than the requirement of SNR, FOCT will not be able to normally calculate the measured current and generate a fault alarm. The modulation signal attenuation can be avoided connecting capacitors at CMB to stabilize the modulation voltage.

4.4. Influences of Measured Current. It can be derived from equation (10) that the second harmonic component fluctuates when the Faraday phase shift changes with the 


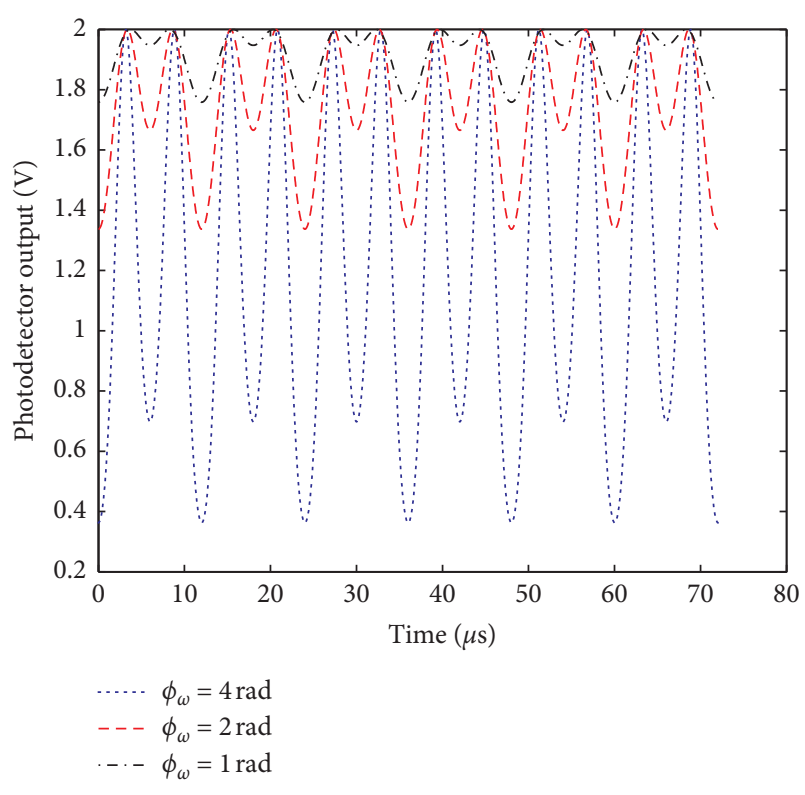

FIGURE 5: Comparison of the photodetector output under different modulation signal amplitudes.

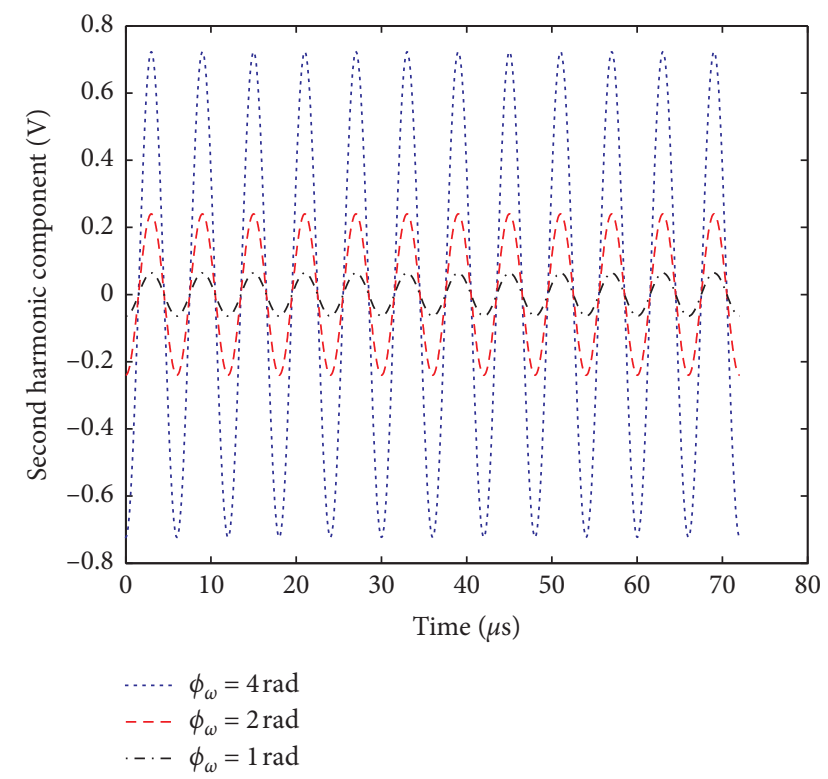

FIgURE 6: Comparison of second harmonic components under different modulation signal amplitudes.

measured current. In order to analyze the influences of measured current on the output of FOCT photodetector, the output signal of the photodetector and second harmonic components are calculated when the measured current is $2000 \mathrm{~A}$ and $10000 \mathrm{~A}$, respectively, and the results are shown in Figure 7.

The Faraday phase shift $\Delta \theta$ is $0.192 \mathrm{rad}$ and $0.96 \mathrm{rad}$ when the measured current $I$ is $2000 \mathrm{~A}$ and $10000 \mathrm{~A}$, respectively. It can be seen from Figure 7 that the value of the photodetector output decreases when the measured current increases. It is $0.39 \mathrm{~V}$ when $I$ is $2000 \mathrm{~A}$, which is

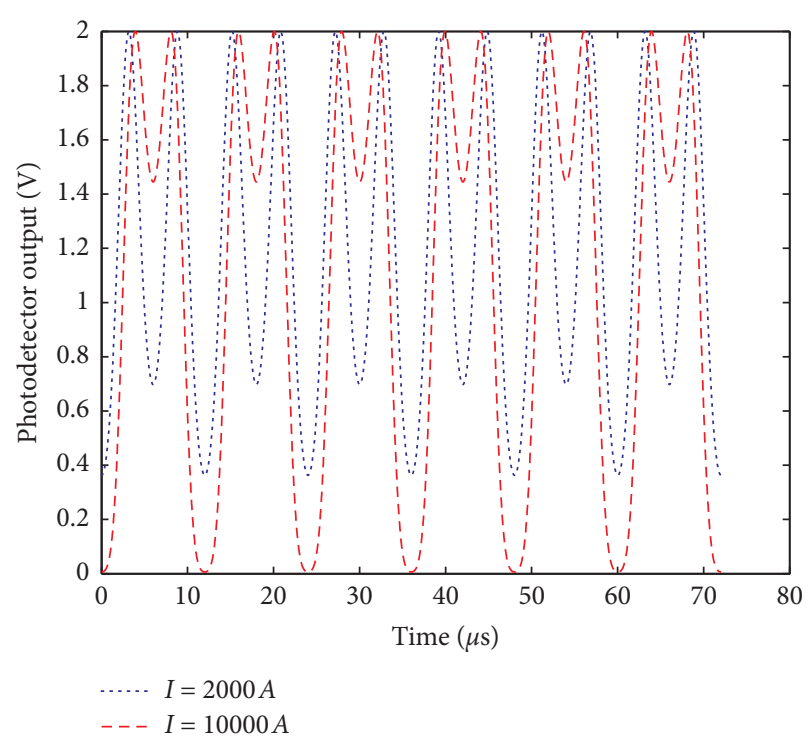

Figure 7: Comparison of the photodetector output under different measured currents.

$0.5 K L P_{0}\left\{1+\cos \left[\Delta \theta+\phi_{e}\right]\right\}$. However, the groove of the photodetector output rises as measured current increases, which is caused by the increase of $\Delta \theta$ and $0.5 K L P_{0}\left\{1+\cos \left[\Delta \theta-\phi_{e}\right]\right\}$.

The peak value of the second harmonic component in Figure 8 is $0.7 \mathrm{~V}$ and $0.4 \mathrm{~V}$ when the measured current is $2000 \mathrm{~A}$ and $10000 \mathrm{~A}$, respectively, which indicates that the second harmonic component decreases as the measured current rises. This is because $\cos \Delta \theta$ decreases with the increase of $\Delta \theta$. Therefore, the increase of the measured current will also lead to the reduction of the second harmonic component, which may lead to FOCT measurement fault when the second harmonic component is smaller than the requirement of SNR. In such occasion, it is necessary to reduce the number of sensing loops to achieve the measurement of large current.

\section{Experimental Verification}

5.1. Overall Design of Experiment. According to the theoretical analysis results, the fault simulation of FOCT is carried out by experiment and the overall design of the experiment is shown in Figure 9. Due to the limitation of the output current amplitude of DC current source, only the influencing experiment of optical path and modulation circuit parameters was carried out. In Figure 9, FOCT is mainly composed of the electronic unit in the measurement interface cabinet, the cable management box (CMB) located in the switch field, and the fiber optical sensor head, which are connected by the cable and optical fiber. The modulator is located at the base of the fiber optical sensor head, and its modulation signal is led out by the electronic unit, transmitted by modulated signal cable to the $\mathrm{CMB}$, and finally connected to the base of the sensor head. In order to simulate the change of optical path and modulation circuit parameters, and further analyze their influencing mechanism on FOCT fault, the optical path attenuator and modulation 


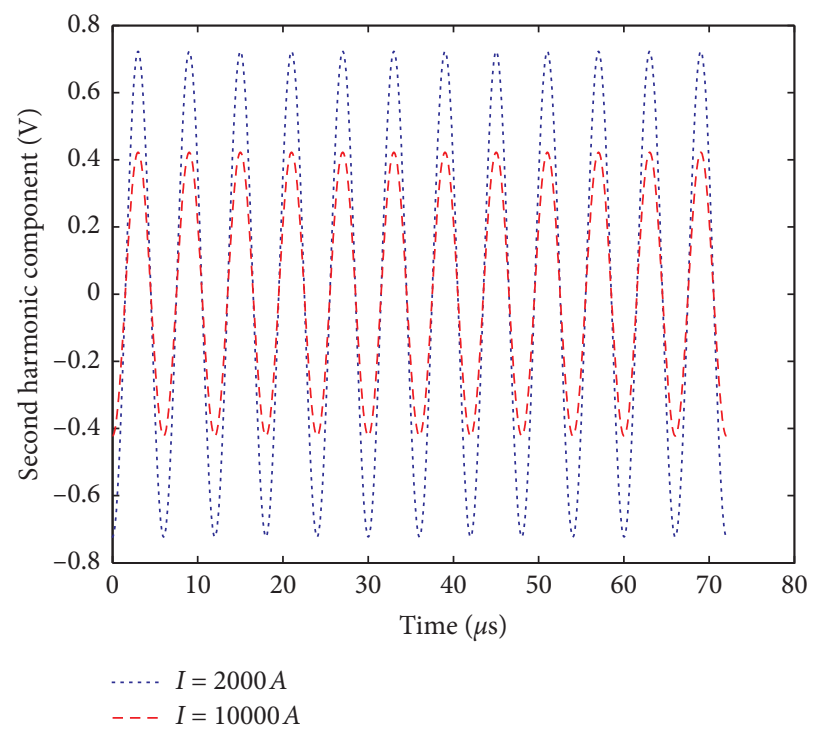

FIGURE 8: Comparison of second harmonic components under different measured currents.

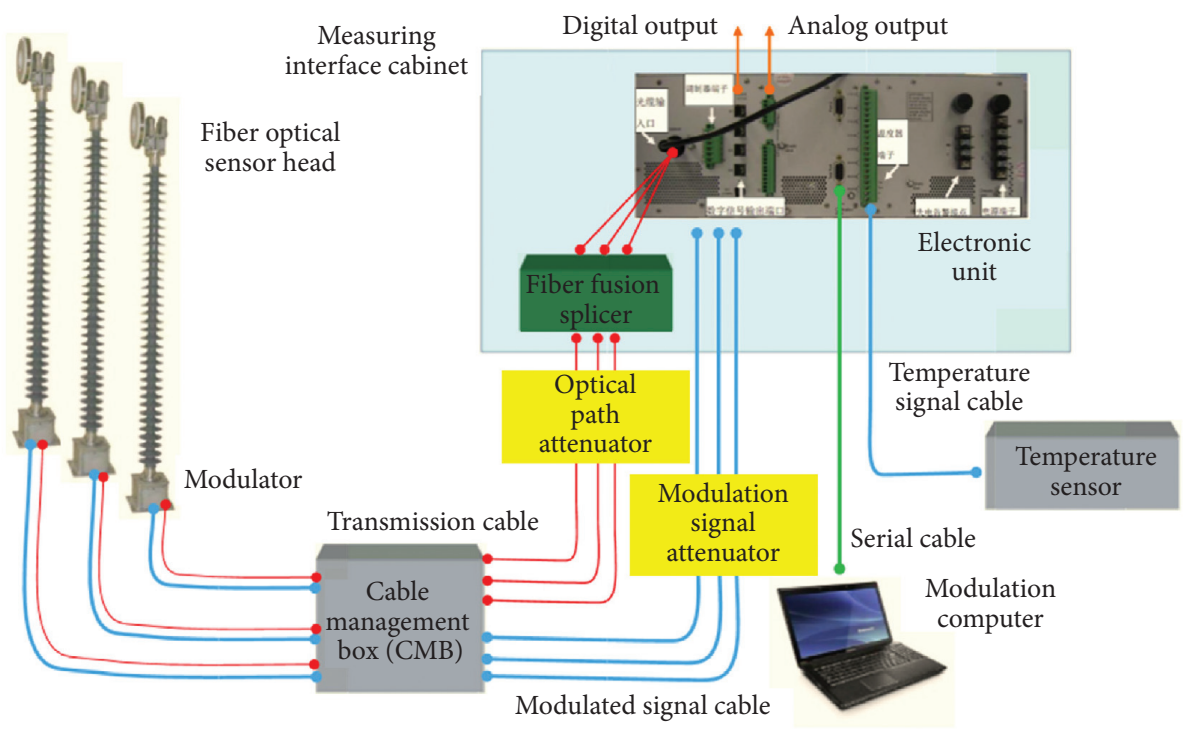

Figure 9: Overall design of the experiment.

signal attenuator are added to the optical path and modulation circuit, respectively. By adjusting the attenuation of the two, the change of optical path and modulation circuit parameters are realized.

The peak value of the FOCT photodetector output is about $1.7 \mathrm{~V}$, and the amplitude of the modulation signal is $4.5 \mathrm{~V}$. The other parameters such as the length of optical fiber, the angular frequency of modulation signal, and the number of sensing loops are consistent with the simulation data in Section 4. Figure 10 is the field wiring diagram of the experiment. For the convenience of wiring, the optical path attenuator and modulation signal attenuator are connected to the optical path and modulation signal transfer circuit in CMB. The optical path attenuator achieves the attenuation of the optical path by changing the light intensity, and the modulation signal attenuator has an adjustable resistance which changes the modulation signal change by turning the load of the whole circuit.

The specific working process of the experiment is as follows: the DC current source provides DC current for the test. When the system is in a stable and normal working state, the optical path attenuator and the modulation signal attenuator are respectively used to realize the parameter attenuation of the two circuits. The working state parameters of FOCT are monitored in real time by the computer connecting electronic unit, and the change of the FOCT photodetector output and its second harmonic component are recorded in case of fault alarm.

5.2. Simulation Results of Optical Path Parameters. In the simulation test of optical path parameters, the 


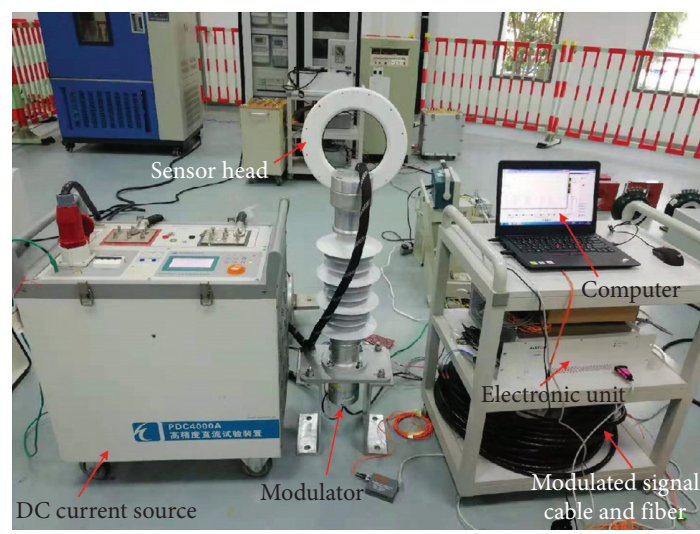

(a)

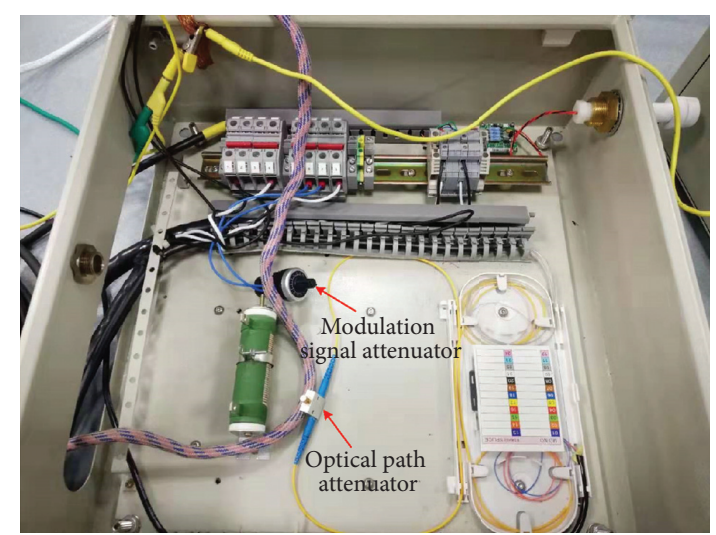

(b)

Figure 10: Field wiring diagram of the fault simulation test. (a) Layout of the experiment. (b) Internal layout of CMB.

attenuation degree of the optical path attenuator is monitored by using an optical power meter. Under normal working condition, the optical power of the path is $150 \mu \mathrm{W}$. In order to simulate the optical path fault, the optical path attenuator is adjusted to reduce the power to $30 \mu \mathrm{W}$, and the FOCT fault indicator light is on. The photodetector output and the second harmonic signal under normal and fault conditions are collected and recorded, respectively. The results are shown in Figures 11 and 12 .

It can be seen from Figure 11 that the peak value of the photodetector output is about $1.7 \mathrm{~V}$ in normal condition, and when the optical power of the system is reduced to $30 \mu \mathrm{W}$ in fault condition, the peak value of the photodetector output is reduced to $0.4 \mathrm{~V}$, indicating a attenuation ratio similar to that of optical power reduction. This shows that the parameters of the light intensity in the optical path are in direct proportion to the amplitude of the photodetector output and verifies that the decrease of the light intensity will lead to the decrease of the photodetector output in the same proportion.

Figure 12 is the comparison diagram of the second harmonic component in normal and fault conditions after optical power attenuation, and the peak value of the second harmonic component is reduced from $0.62 \mathrm{~V}$ to $0.13 \mathrm{~V}$. According to the description of FOCT working parameters provided by the manufacturer, a fault alarm will appear when the peak value of the second harmonic component is lower than $0.36 \mathrm{~V}$. Therefore, FOCT is in fault condition when the optical power is reduced to $30 \mu \mathrm{W}$.

\subsection{Simulation Results of Modulation Circuit Parameters.}

The simulation of modulation circuit parameters is realized by reducing the modulation signal amplitude of FOCT. Under normal condition, the modulation signal amplitude of FOCT is $4.5 \mathrm{~V}$. The modulation signal attenuator is

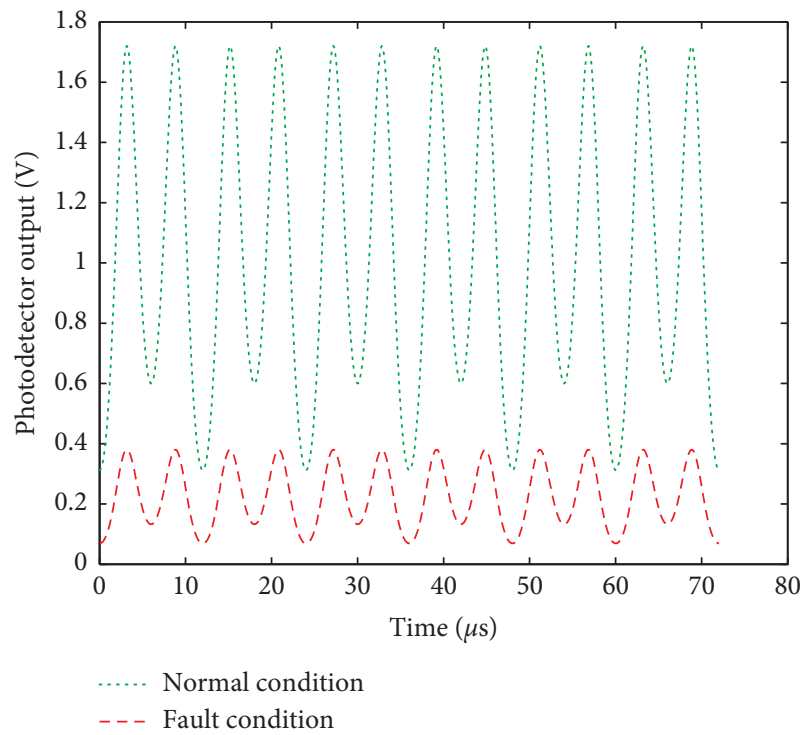

Figure 11: Comparison diagram of the detector output signal under optical power attenuation.

adjusted to reduce the amplitude to $2.25 \mathrm{~V}$ applied to the modulator through voltage division, and thus, the FOCT fault alarm light is on. Figures 13 and 14 show the photodetector output and the second harmonic component under different modulation signal amplitudes.

As shown in Figure 13, the amplitude of the detector output signal fluctuates from $0.3 \mathrm{~V}$ to $1.7 \mathrm{~V}$ in normal condition. When the modulation signal is reduced to half of the normal condition, the peak value of the photodetector output remains unchanged, while its minimum value rises to $1.15 \mathrm{~V}$, which shows that the decrease of the modulation signal will reduce the modulation factor and further lead to the increase of the minimum value of the photodetector output as proved in the simulation results in Section 4.3. 


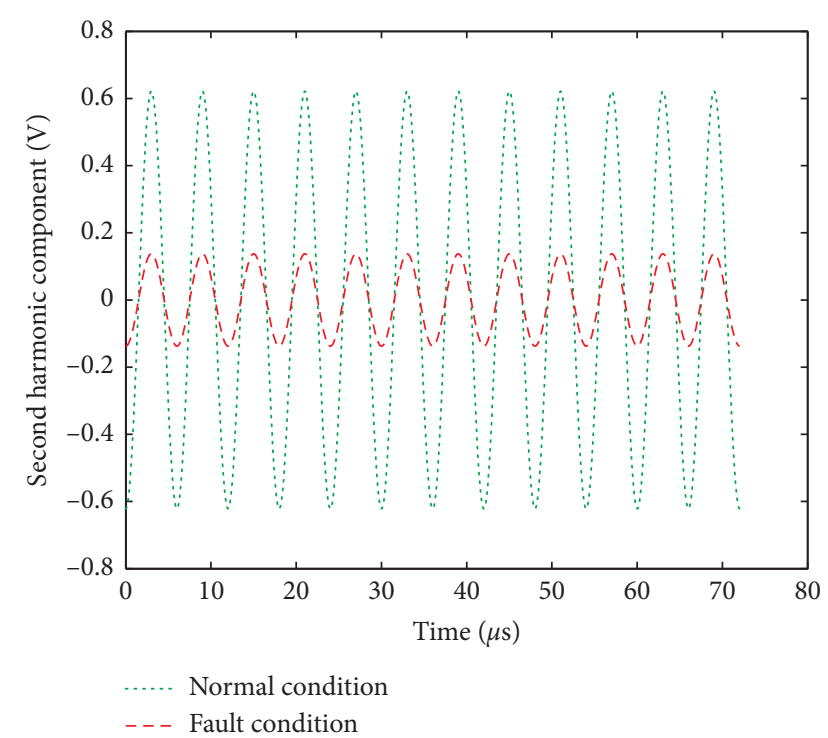

Figure 12: Comparison diagram of the second harmonic component under optical power attenuation.

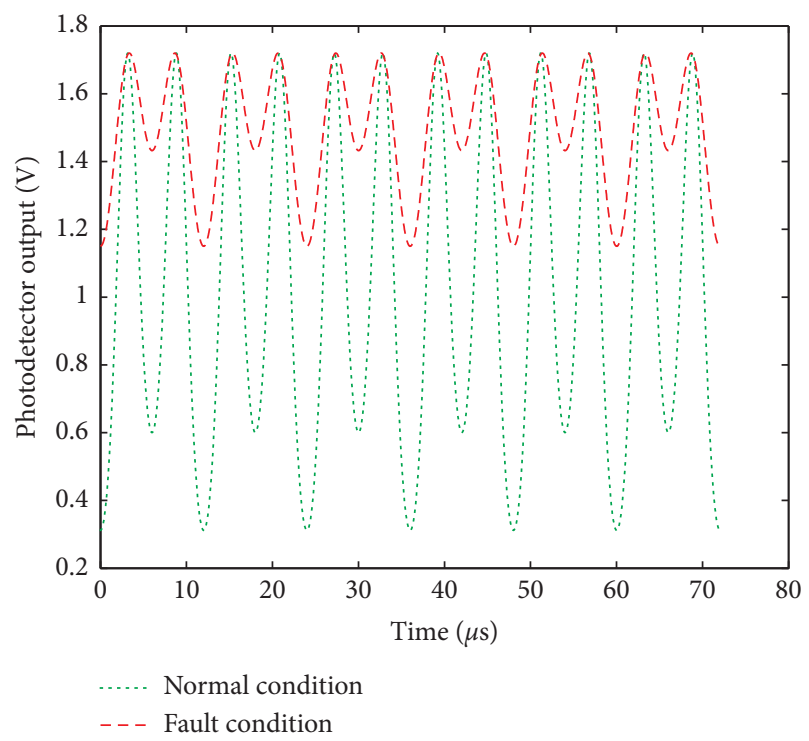

FIgURE 13: Comparison diagram of the photodetector output under modulation signal attenuation.

When the modulation voltage is reduced to half of the normal condition value, the peak value of the second harmonic component is reduced from $0.6 \mathrm{~V}$ to $0.2 \mathrm{~V}$, as shown in Figure 14. This is because the reduction of the modulation signal causes the modulation factor to decrease in the same proportion, from $2.33 \mathrm{rad}$ in normal condition to $1.165 \mathrm{rad}$ in fault condition. It can be seen from Figure 4 that when the modulation factor is less than $3.1 \mathrm{rad}$, the reduction of the modulation factor will cause the rapid decrease of the second harmonic component, and then, FOCT will send a fault alarm when the second harmonic component is lower than the normal parameter range.

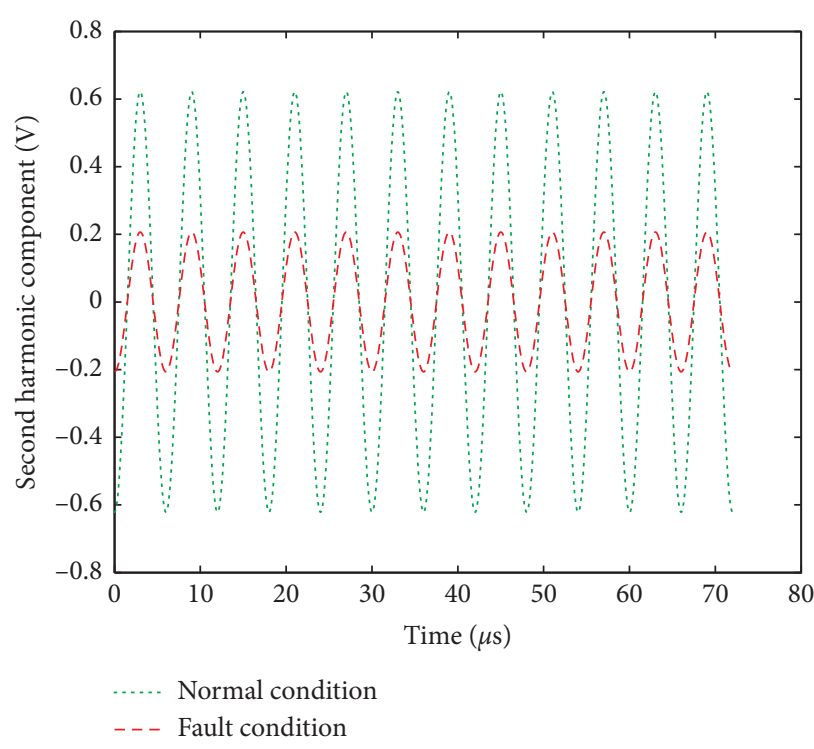

FIgURE 14: Comparison diagram of the second harmonic component under modulation signal attenuation.

\section{Conclusion}

In order to investigate the fault mechanism of FOCT with sine wave modulation, the photodetector output model is established and the signal processing for the calculation of measured current is further studied in this paper. Theoretical analysis indicates that the reduction of the second harmonic component in the photodetector output is the root cause of FOCT fault, and the parameters of the optical path, modulation circuit, and measured current change the working state of FOCT by affecting the second harmonic amplitude, and the influences of three main parameters on the photodetector output and second harmonic component are analyzed by simulation, and the results show that the parameters of the optical path are directly proportional to the photodetector output and second harmonic component, while the decrease of modulation signal amplitude reduces the dynamic range of photodetector output signal and further reduces the second harmonic component. The increase of measured current reduces the second harmonic component by increasing the Faraday phase shift. The simulation experiment of the optical path and modulation circuit parameters verifies the correctness of the theoretical analysis, and the research results can be used for troubleshooting and reliability improvement analysis of FOCT.

\section{Data Availability}

The data used to support the findings of this study are available from the corresponding author upon request.

\section{Conflicts of Interest}

The authors declare no potential conflicts of interest with respect to the research, authorship, and/or publication of this article. 


\section{Acknowledgments}

The project was supported by State Grid Jiangsu Electric Power Co., Ltd. (J2020012, Research on fault mechanism and reliability improvement technology of fiber optical current transformer).

\section{References}

[1] Y. Wu and J. Guo, "Typical fault overvoltage characteristics of \pm 1100 kV DC filter," Electrical Power Engineering Technology, vol. 39, no. 1, pp. 130-137, 2020.

[2] Z. Li, X. Xiang, and T. Hu, "An improved digital integral algorithm to enhance the measurement accuracy of Rogowski coil-based electronic transformers," International Journal of Electrical Power \& Energy Systems, vol. 118, Article ID 105806, pp. 1-8, 2020.

[3] H. Zhai, Z. Li, and H. Huang, "Terminal location selection study for UHVDC hierarchical connection to power grid," Electrical Power Engineering Technology, vol. 37, no. 1, pp. 65-71, 2018.

[4] X. Liang, P. Zhang, and Y. Chang, "Recent advances in highvoltage direct-current power transmission and its developing potential," Power System Technology, vol. 36, no. 4, pp. 1-9, 2012.

[5] K. Bohnert, C.-P. Hsu, L. Yang, A. Frank, G. M. Muller, and P. Gabus, "Fiber-optic current sensor tolerant to imperfections of polarization-maintaining fiber connectors," Journal of Lightwave Technology, vol. 36, no. 11, pp. 2161-2165, 2018.

[6] X. Guo, Y. Zhou, and N. Mei, "Construction and characteristic analysis of Zhangbei flexible DC grid," Power System Technology, vol. 42, no. 11, pp. 3698-3708, 2018.

[7] J. Zhang, Y. Hu, and F. Liu, "Critical technology of on-site calibration system for DC electronic instrument transformer," High Voltage Engineering, vol. 42, no. 9, pp. 3003-3010, 2016.

[8] S. Cheng, G. Zhang, and Z. Guo, "Influence mechanism of conductor eccentricity on all-fiber optic current transformer," Automation of Electric Power Systems, vol. 39, no. 13, pp. 137-143, 2015.

[9] Q. Zhang, H. Li, and H. Cheng, "Research on online monitoring technology for active electronic current transformer," High Voltage Engineering, vol. 42, no. 1, pp. 208-213, 2016.

[10] L. Wang, G. Chen, J. Ji, J. Sun, J. Qian, and X. Liu, "Methods of fault diagnosis in fiber optic current transducer based on Allan variance," Mathematical Problems in Engineering, vol. 2014, Article ID 831075, 6 pages, 2014.

[11] Y. Wang, Z. Wang, and S. Sun, "Effect of temperature characteristic of Faraday rotator on passively demodulated alloptical fiber current transformers," Power System Technology, vol. 37, no. 1, pp. 206-210, 2013.

[12] K. Sasaki, M. Takahashi, and Y. Hirata, "Temperature-insensitive sagnac-type optical current transformer," Journal of Lightwave Technology, vol. 33, no. 12, pp. 2463-2467, 2015.

[13] H. Luan, W. Yin, and F. Zhou, "A fast recognition strategy for abnormal transformer data in DC protection and control device," Power System Technology, vol. 42, no. 12, pp. 1-7, 2018.

[14] F. Pang, Y. Liu, and Y. Yuan, "Influencing factors analysis on the detector output signal of fiber optic current transformer with sine modulation," Measurement, vol. 151, Article ID 107151, pp. 1-8, 2020.

[15] K. Ouyang, X. Hong, and J. Wu, "A novel method on modulation and demodulation of all fiber optical current transformer," Chinese Journal of Lasers, vol. 42, no. 1, pp. 1-6, 2015.
[16] H. Wang, Y. Guan, and C. Hu, "Temperature error of fiber optic current transformer," Electric Power Automation Equipment, vol. 237, no. 12, pp. 200-204, 2017.

[17] C. Zhang, C. Zhang, and X. Wang, "Signal processing system for digital closed-loop fiber optic current sensor," Proceedings of the CSEE, vol. 29, no. 30, pp. 42-46, 2009.

[18] J. Li, Z. Li, and D. Zhang, "Open-loop detection of fiber optic current transducer," Infrared and Laser Engineering, vol. 43, no. 5, pp. 1648-1653, 2014. 